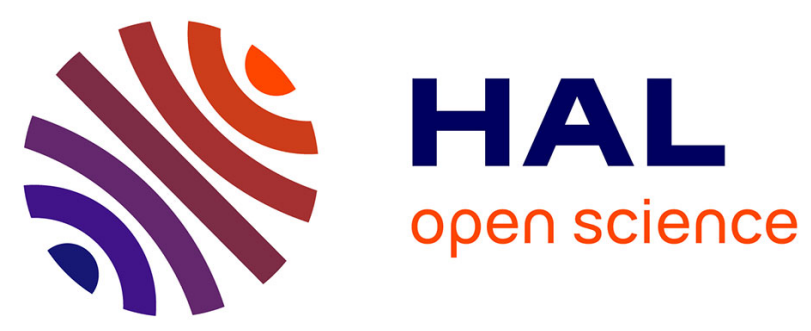

\title{
Deterioration estimation for predicting and controlling RUL of a friction drive system
}

Diego Rodriguez Obando, John Jairo Martinez Molina, Christophe Bérenguer

\section{To cite this version:}

Diego Rodriguez Obando, John Jairo Martinez Molina, Christophe Bérenguer. Deterioration estimation for predicting and controlling RUL of a friction drive system. ISA Transactions, 2021, 113, pp.97-110. 10.1016/j.isatra.2020.10.013 . hal-02969131

\section{HAL Id: hal-02969131 \\ https://hal.science/hal-02969131}

Submitted on 10 May 2021

HAL is a multi-disciplinary open access archive for the deposit and dissemination of scientific research documents, whether they are published or not. The documents may come from teaching and research institutions in France or abroad, or from public or private research centers.
L'archive ouverte pluridisciplinaire HAL, est destinée au dépôt et à la diffusion de documents scientifiques de niveau recherche, publiés ou non, émanant des établissements d'enseignement et de recherche français ou étrangers, des laboratoires publics ou privés.

\section{(ㅇ)(1) $\$$}

Distributed under a Creative Commons Attribution - NonCommercial - NoDerivatives| 4.0 


\title{
Deterioration estimation for predicting and controlling RUL of a friction drive system
}

\author{
Diego Rodriguez Obando, John J. Martinez, Christophe Bérenguer \\ Univ. Grenoble Alpes, CNRS, Grenoble INP, GIPSA-lab, 38000 Grenoble, France
}

\begin{abstract}
This paper develops a method for estimating the state of deterioration of a friction drive system and presents its use for predicting and controlling the Remaining Useful Life (RUL) of such a system. The friction drive system is assumed to be affected by endogenous uncertainties and exogenous disturbances. The proposed method is intended for on-line estimation of the contact surface deterioration and it is based on a parameter-varying model that includes both the motion dynamics and the deterioration dynamics of the device. Since, in the presented setting, the control actions on the mechanical system play a role on the non-linear deterioration dynamics, an Extended Kalman Filter is developed for simultaneously estimating both the state of deterioration and its associated estimation error bounds. A numerical example is presented to illustrate the interest of such estimations for RUL prognosis and RUL control. The presented example considers the availability of angular speed measurements and the possibility of re-planing motor torques and/or re-planing desired angular speeds in order to control RUL based on RUL prognosis.
\end{abstract}

Keywords: Friction drive systems, remaining useful life, prognosis, condition monitoring, nonlinear observers, parameter-varying systems.

\section{Introduction}

When addressing the Prognostics and Health Management (PHM) of a mechatronic system, one of the main issue concerns the adaptation of the system's behavior according to its current estimated reliability and its predicted residual life. These adaptations are intended for increasing the ability of the system to accomplish a mission, with a high level of performance, low energy consumption, and for decreasing the operational costs. The latter are directly related to the associated maintenance costs and lifetime of the system components. Intelligent mechatronic systems often comprise a controlled system and the controller can be used for modifying their behavior in order to reach a 
predefined lifetime and to trade-off with performance. Such systems can be operated in a reliableadaptive way for maintenance planning in order to reduce operational costs, as presented in [1] [2]. Thus, control-loop adaptations could be part of the solution of a multi-objective optimization problem concerning both short-term (i.e. performance) and long- term (i.e. lifetime and durability) objectives, most of them being conflicting objectives, for instance, maximizing productivity and avoiding premature wearing of the system components.

Within the context of this post-prognosis decision-making issue [3], the prediction of the Remaining Useful Life (RUL) of a device is therefore a key issue for solving such multi-objective optimization and control problem. The information on the predicted RUL is not only necessary to verify whether the mission goal can be accomplished, but it is also important to aid in online decision-making activities such as fault mitigation, control reconfiguration $[4,5,6]$, mission replanning $[7,8][9]$ and eventually maintenance operations. Mission replanning can be implemented using for example load-sharing mechanisms at different levels [10], i.e between components within a multi-component system or between equipments within a fleet, whereas control reconfiguration can be achieved by a suitable modification of desired control-loop references for the operation of a component or an item, as stated in [11]. However, eventually, both problems of mission replanning and controller reconfiguration can be considered as a RUL control problem formulated as a multi-objective optimization problem.

In this paper, we aim at tackling the multi-objective RUL optimization problem as a control problem. To this aim, it is first necessary to build a model that links the control inputs of the considered system both to its performance level and to its deterioration behavior, which are often two antagonistic processes. In $[11,12]$, it is assumed that the control input is seen as a source of stress deteriorating the actuator. This assumption is in general accepted by engineers in several applications. However, there are some works that consider dissipated energy as an image of the heat and material worn in railway wheel wearing [13].

The dissipated energy producing wear can be related to control-loop decisions, as it is presented in this work. Mechatronic controlled systems are in general affected by endogenous uncertainties and exogenous disturbances that make deterioration a random variable, and therefore the RUL prediction and control become more difficult without the inclusion of a suitable characterization of the uncertainty on the current deterioration [9]. Such an uncertainty could be a time-varying 
uncertainty ; it is therefore required to track uncertainties in real-time in order to update both the prediction and the control of the RUL. The role of the online estimation of the current deterioration is very important within this context and turns out to be a key enabler for the implementation of the full PHM chain, since these estimations will be used as initial conditions for predicting RUL. Hence, especially if poorly characterized, a high uncertainty on the state of deterioration will provide poor RUL predictions and by consequence poor RUL control performances.

Within this context of PHM and post-prognosis decision making for optimal RUL control, the direct contribution of the paper is twofold:

1. First, we develop a method for estimating the state of deterioration of a mechanical system using external measurements only, with application to a friction drive system. The deterioration estimation and its uncertainties are then used for predicting the Remaining Useful Life (RUL) of the considered system. The friction drive system is assumed to be affected by endogenous uncertainties (e.g. unknown and possible time-varying parameters) and exogenous disturbances (e.g. the system load, the operating point changes, sensor noise and unpredictable input disturbances). The proposed method is intended for on-line estimation of contact surface deterioration and it is based on a parameter-varying model that includes both the motion dynamics and the deterioration dynamics of the device. The deterioration is modeled here as a function of the energy losses (at the contact level) during the system operation and this explicit physics-based modeling of the deterioration permits the estimation of the deterioration using the measurements of physical variables. One of the difficulty within a PHM context comes from the fact that such a deterioration cannot be measured directly and has to be estimated on-line using external measurements only, [14, 15]. To this aim, we propose a generic approach (i.e. valid not only for friction drive system) relying on an extended model developped to jointly capture the process' own dynamics and the effects of the deterioration on it. The links between deterioration and process dynamics can be identified by the physics of the phenomena involved. The deterioration changes the dynamics of the process, and thanks to this extended model, it becomes possible to build an estimator of deterioration, using only external, readily available measurements. An Extended Kalman Filter is thus developed and implemented for estimating both the state of deterioration and its associated estimation error bounds, under the assumption that the only available mea- 
surements are the angular speeds. The advantage of this proposed method over more classical approaches to deterioration estimation is that we do not assume that a measurement directly or indirectly correlated to the deterioration is readily available, and we develop a modelling approach (as explained above) making it possible to estimate the deterioration online, using external measurements only, simple to implement.

2. Second, we propose an architecture for RUL prognosis and control, that elaborates the control decision using the online estimated deterioration with its associated uncertainties. Thanks to the used physics-based deterioration model, the approach for the RUL control is based on the possibility of acting on physical variables (e.g. re-configuring motor torques and/or making adjustments to the reference inputs, i.e. desired angular speed) to modify the deterioration behavior, which in turn allows to control indirectly the RUL in closed-loop, based on RUL prognosis information, [5]. Very often, in PHM approaches, one seeks to make decisions (e.g. maintenance decisions) to adapt at best to the estimated RUL, and does not try to control it. The originality of our proposed architecture is to seek to control this RUL by modifying the way the system is operated, which opens new perspectives in post-prognosis decision-making.

Beyond these direct contributions, the framework proposed in this paper can also be seen as first methodological step towards the development of an integrative scheme for deterioration estimation, RUL prognosis and RUL control, based on "external", directly and easily available monitoring information, i.e., in the present case, angular speeds. These two contributions (procedure for the estimation of the deterioration and architecture for RUL prediction and control) constitute together an original example of an integrated scheme for the synthesis of a health-aware controlled system. Because it does not rely on the -often oversimplifying- assumption that the system deterioration can be directly measured, the proposed scheme gains in applicability. As such, the main contribution of the model is the proposed methodological framework and integrative scheme, rather than the resulting model itself, developed for the special case of a friction drive system.

This paper is organized as follows: Section 2 presents some preliminaries and working assumptions concerning the description of the friction drive system and the modeling of its deterioration dynamics. Section 3 states the tackled problem in terms of deterioration estimation, and RUL prediction and control. Section 4 presents the proposed method for deterioration estimation based on an extended model and using an Extended Kalman Filter. Section 5 presents an architecture 
for predicting and controlling the RUL based on the previous proposed deterioration estimator. In Section 6, a numerical example is presented, which illustrates the proposed methodology. Finally, Section 7 draws the main conclusions of this work and gives some perspective for future developments.

\section{Acronyms \& Notations}

PHM prognostics and health management

EKF $\quad$ extended Kalman filter

RUL remaining useful life

EoL end of life (of the system)

$A, B, D \quad$ matrices of the state-space representation of the system dynamics

$\alpha$

$B_{1}$ contact quality coefficient

$B_{2}$ viscous damping coefficient of the driver device

$D$ viscous damping coefficient of the driven device contact surface deterioration $D_{a}^{\max }$ maximal admissible value of deterioration unknown input used in the deterioration process model (Gaussian process noise)

$\eta_{1}$ (resp. $\eta_{2}$ ) measurement noise for sensor 1 (resp. 2)

$\boldsymbol{F}$

$F_{c}$

$\boldsymbol{H}(\boldsymbol{x}), \boldsymbol{B}, \boldsymbol{E}(\boldsymbol{x}), \boldsymbol{C}$

$J_{1}$

$J_{2}$

$K$

$\mathcal{L}$

$m$

$\omega_{1}$

$\omega_{2}$
Jacobian of the function $\boldsymbol{H}(\boldsymbol{x})$

contact force

matrices of the non-linear state space representation of the augmented system

moment of inertia of the driver device

moment of inertia of the driven device

Kalman gain

objective cost function

coefficient of the linear model used for $\alpha$

angular speeds of the driver device

angular speeds of the driven device 


$\begin{array}{ll}P_{c} & \text { dissipated power at the contact level } \\ \boldsymbol{P} & \text { estimation error covariance matrix } \\ Q & \text { covariance matrix of the process Gaussian noise } \varepsilon \\ R & \text { covariance matrix of the measurement Gaussian noise } \eta \\ r_{1} & \text { radius of the driver device } \\ r_{2} & \text { radius of the driven device } \\ \boldsymbol{S} & \text { innovation covariance matrix } \\ \boldsymbol{\theta} & \text { decision vector } \\ T_{m} & \text { motor torque } \\ T_{L} & \text { load torque seen from the driver side } \\ T_{S} & \text { source of torque observed from the driven side } \\ t_{s} & \text { sampling period } \\ u & \text { system input } \\ \boldsymbol{x} & \text { state vector of the augmented system } \boldsymbol{x}=\left[\omega_{1}(t) \omega_{2}(t) \alpha(t) m(t) D(t)\right]^{\top} \\ y & \text { system output }\end{array}$

\section{Preliminaries}

\subsection{Description of the studied friction drive system $\mathcal{E}$ modelling assumptions}

In this paper, the studied system concerns a roller-on-tire actuator, as it is depicted in Fig. 1. This is a friction drive system composed by a driver device (a dc motor) and a driven device (a wheel).

Both devices are affected by a contact force, denoted $F_{c}$, that is produced by the driver device and causes a torque which drives the driven device. Because the studied system concerns a rolling friction process, it is assumed that the contact force only depends on the tangential speeds of both devices (i.e. neglecting the pre-sliding friction and the Stribeck effect, but keeping the viscous component of friction forces). Thus the contact force $F_{c}(t)$ will be proportional to the relative tangential speed at the contact level, as follows:

$$
F_{c}=\alpha\left(r_{1} \omega_{1}-r_{2} \omega_{2}\right)
$$

where $\alpha \geq 0$ is a scalar named here as the contact quality coefficient, which is considered an uncertain parameter. The symbols $\omega_{1}$ and $\omega_{2}$ stand for the angular speeds of the driver device and 


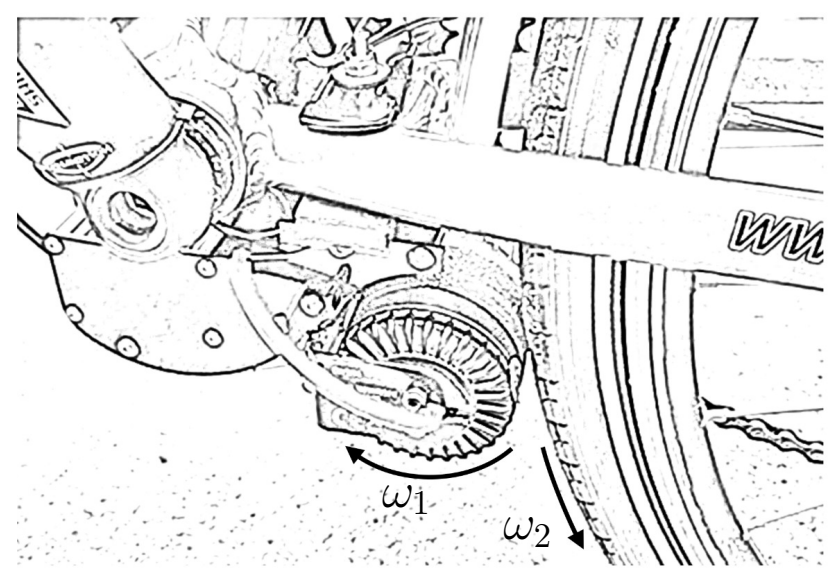

Figure 1: Example of a friction drive system : A roller-on-tire actuator for an electrical bike.

driven device, respectively. $r_{1}$ and $r_{2}$ are constant values concerning the radius of the driver device and the driven device, respectively. Contact forces in dynamical friction models for estimation and control are largely studied into the literature, see for instance [16]. The contact force (1) has been derived from the LuGre model presented in [17] and [18] by keeping only the viscous dissipation related terms.

Using Newton's laws of motion, the friction drive dynamics can be written as follows (see also $[19])$ :

$$
\begin{aligned}
& J_{1} \dot{\omega}_{1}=T_{m}+T_{L}-B_{1} \omega_{1} \\
& J_{2} \dot{\omega}_{2}=T_{S}-B_{2} \omega_{2}
\end{aligned}
$$

where $T_{m}$ is the motor torque. $B_{1} \omega_{1}$ and $B_{2} \omega_{2}$ are torques caused by the viscous damping of the driver device and the driven device, respectively. $J_{1}$ and $J_{2}$ are the moments of inertia of the driver device and of the driven device, respectively.

$T_{L}$ is a load torque seen from the driver side, whereas $T_{S}$ is a source of torque observed from the driven side. Both torques can be written in terms of the contact force $F_{c}$ as follows:

$$
\begin{aligned}
& T_{L}(t)=-F_{c} r_{1} \\
& T_{S}(t)=F_{c} r_{2}
\end{aligned}
$$




\subsection{Dissipation-energy based model of deterioration}

The dissipated power at the contact level can be computed as follows:

$$
P_{c}(t)=F_{c}\left(r_{1} \omega_{1}-r_{2} \omega_{2}\right)=\alpha p(\omega)
$$

where $p(\omega)$ denotes a non-linear scalar valued function of speeds $\omega_{1}$ and $\omega_{2}$. That is,

$$
p(\omega)=\left(r_{1} \omega_{1}-r_{2} \omega_{2}\right)^{2}
$$

In the sequel, $\omega$ denotes a column vector whose components are the speeds $\omega_{1}$ and $\omega_{2}$.

In the presented setting, the resulting dissipated energy is considered as an image of the heat and the material worn at the contact level during traction. This assumption is consistent with the Archard's equation that is commonly used in railway industry for wear prediction (see for instance [20] and [13]). Thus, the contact surface deterioration, denoted $D(t)$, is defined here as the dissipated energy at the contact level:

$$
D(t)=\int_{0}^{t} P_{c}(t) d t=\int_{0}^{t} \alpha\left(r_{1} \omega_{1}-r_{2} \omega_{2}\right)^{2} d t
$$

Note that, from (8), the time-derivative of $D$ can be written as follows:

$$
\dot{D}(t)=P_{c}(t)=\alpha\left(r_{1} \omega_{1}-r_{2} \omega_{2}\right)^{2}=\alpha p(\omega)
$$

which provides a dynamical equation of the deterioration that will be used later.

By considering the system deterioration as a measure of the loss in the actuator ability to transfer mechanical power to the load device, an additional assumption is that the contact quality coefficient $\alpha(t)$, with initial value $\alpha(0)$, monotonically decreases as $D(t)$ increases. That is,

$$
\alpha(t)=-m D(t)+\alpha(0)
$$

where $\alpha(0)$ is considered to be an unknown constant parameter, whereas $m \geq 0$ could be considered as an unknown time-varying parameter. When compared to classical phenomenological degradation processes often considered in reliability, [21], one of the interest of the deterioration model summarized by Equation (10) is that it provides a physical meaning to the deterioration behavior, which will allow in turn both to estimate the deterioration (not directly measurable) from external physical measurements and to identify the possible actions on physical variables within the system to control the deterioration. This deterioration model is thus one of the key feature allowing to 
develop the integrative scheme "from deterioration estimation to RUL control" mentioned earlier in the paper. This section focuses on modeling the deterioration dynamics, however Equation (10) could also describes system improvements, by considering $m<0$.

Using (10) one can compute the time-derivative of $\alpha$, as $\dot{\alpha}(t)=-m \dot{D}-\dot{m} D$. Now, consider that

$$
\dot{m}=\varepsilon
$$

which means that the parameter $m$ could change during the lifetime of the system and its changes are caused for an unknown input denoted $\varepsilon$. Hence:

$$
\dot{\alpha}=-m p(\omega) \alpha+D \varepsilon
$$

Remark, from (9) and (10), that the deterioration $D \geq 0$ is bounded. In addition, from (10) its maximal value can be a priori calculated as $D=\frac{\alpha(0)}{m}$, when $\alpha=0$.

\subsection{Summary of the considered modeling assumptions}

\subsubsection{Mechanical model assumptions:}

The mechanical model is built based on the main following assumptions :

- it is assumed that the angular speeds of the driver device and driven device are both measured by noisy sensors ;

- the motor torque is known at any given time. A motion controller uses this torque to control the driven device speed ;

- it is assumed that the contact force only depends on the tangential speeds of both devices, i.e. the pre-sliding friction and the Stribeck effect are neglected but the viscous component of friction forces is kept, as it is considered that only this component of the friction forces dissipates energy and then produces deterioration. The contact forces depends on a contact quality coefficient $\alpha \geq 0$;

- it is considered that there is no available measurement for the contact quality coefficient $\alpha$. This parameter decreases when the deterioration increases. $\alpha$ is considered as an uncertain parameter that has to be estimated. 


\subsubsection{Deterioration dynamics assumptions:}

The following main assumptions are made on the deterioration dynamics:

- There are no available measurements for $m$ and $D$. These two variables are used for modeling the decay of the contact quality coefficient $\alpha$ which starts at $\alpha(0)>0$ (as presented in (10)). The parameter $m$ allows relating the contact quality coefficient $\alpha$ to the deterioration $D$;

- the deterioration $D$ has a physical interpretation (i.e. it corresponds to the dissipated energy at the contact level), and by construction this energy is bounded (i.e. the component can not deteriorate indefinitely) ;

- the parameter $m$ is here considered as a positive unknown parameter (positive values allow us for modeling deterioration). This parameter could be a time-varying parameter and its dynamics is simply modeled as $\dot{m}=\epsilon$, which $\epsilon$ could be a random input, such that $m$ remains positive but can exhibit random behaviors ;

- finally, it is considered that speed-sensors noises are more important than deterioration modeling errors.

\section{Problem statement}

Before stating the problem in terms of deterioration estimation, RUL prediction and RUL control, two important definitions are first presented below.

Definition 1. The system deterioration is defined as the loss in the actuator ability to transfer mechanical power to the driven device. A deterioration value $D=0$ means that there is no deterioration of the contact surface, whereas $D=D_{a}, D_{a}>0$, means that deterioration has reached a deterioration value equal to $D_{a}$.

Definition 2. The Remaining Useful Life (RUL) is defined as a prediction of the remaining time that the actuator is able to function in admissible conditions for transmitting mechanical power to the driven load. Hereinafter, RUL will be the remaining time to reach one of the following conditions: i) the parameter $\alpha$ reaches the value $\alpha=0$ or ii) the deterioration $D$ reaches a value 
$D=D_{a}^{\max }$, where $D_{a}^{\max }$ stands for a maximal admissible value of deterioration.

A system is considered as completely deteriorated when one of the following situations is achieved: the deterioration $D$ reaches a given maximal admissible value and/or the parameter $\alpha$ reaches its minimal possible value (which is equal to zero). The former case concerns a premature wear of the contact surface because $\alpha=0$ and $D<D_{a}^{\max }$.

Now, let us represent the friction drive dynamics as an uncertain linear system in the state space representation:

$$
\left[\begin{array}{c}
\dot{\omega}_{1} \\
\dot{\omega}_{2}
\end{array}\right]=\left[\begin{array}{cc}
\frac{-\alpha r_{1}^{2}-B_{1}}{J_{1}} & \frac{\alpha r_{1} r_{2}}{J_{1}} \\
\frac{\alpha r_{2} r_{1}}{J_{2}} & \frac{-\alpha r_{2}^{2}-B_{2}}{J_{2}}
\end{array}\right]\left[\begin{array}{l}
\omega_{1} \\
\omega_{2}
\end{array}\right]+\left[\begin{array}{c}
\frac{1}{J_{1}} \\
0
\end{array}\right] T_{m}
$$

where $\alpha$ concerns an unknown physical time-varying parameter characterizing the deterioration. Using (9), (12) and (11) it is possible to rewrite the deterioration dynamics as follows:

$$
\left[\begin{array}{c}
\dot{\alpha} \\
\dot{m} \\
\dot{D}
\end{array}\right]=\left[\begin{array}{c}
-m \alpha \\
0 \\
\alpha
\end{array}\right] p(\omega)+\left[\begin{array}{l}
D \\
1 \\
0
\end{array}\right] \varepsilon
$$

Clearly, the deterioration dynamics could be driven by the input $p(\omega)$ defined in $(7)$. Considering system (13) as a controllable one (i.e. under the assumption that $\alpha \neq 0$ and its time-derivatives are very slow), the signal $p(\omega)$ can be governed by the motion controller. However, the dynamics (14) is a function of the uncertain parameters $\alpha$ and $m$.

In this work, we suppose the availability of sensors that measure the physical states $\omega$. That is,

$$
y=\omega+\eta
$$

where $\eta$ is a column vector whose components are the measurement noises $\eta_{1}$ and $\eta_{2}$ for sensor 1 and sensor 2, respectively. In addition, we suppose that those noises are random signals with zero mean and known covariance matrix $R$.

Therefore, an estimation of the current deterioration level can be performed by using the available sensors, the information about the manipulable variables and a deterioration model. Now, we 
are ready to state the first problem. The deterioration estimation problem can be stated as follows:

Problem 1. Deterioration estimation problem: At every time instant, estimate the states of the extended system described by dynamics (13)-(14) and, characterize the uncertainties of such estimations, by using the current motor torque $T_{m}$ and the measurements $y$.

Remark that, one of the originality of the paper concerns the estimation of the deterioration dynamics by using an extended dynamical model, equations (13)-(14). This extended dynamical model is suitable for estimation because it is assumed that there are no measurements available for $\alpha, m$ and $D$. In particular, $D$ can not be measured directly, but the extended model would allow its estimation. In addition, the model (14) can be used for predicting the future values of such states if initial conditions of the states and future trajectories of $p(\omega)$ are available. Thus, the RUL prediction problem can be stated as follows:

Problem 2. RUL prediction problem: perform a prediction of possible trajectories of $\alpha, m$ and D, by using dynamics (14), which will be reached for a set of estimated current values of the deterioration states, provided by a deterioration estimator (solution of Problem 1), and future operation conditions, provided by a decision maker system (e.g. expected values of $p(\omega)$ ). Subsequently, compute a set of possible reachable RULs (e.g. mean and variance) from the predicted trajectories of $D$ and $\alpha$.

Remark that the RUL prediction will be affected by the uncertainties on the initial conditions of the deterioration level $D$, the uncertainties on the parameters $\alpha$ and $m$, as well as the uncertainties on the future operation conditions (characterized by the expected values of $p(\omega)$ ). The RUL prediction considered here focuses on estimating the uncertain distribution of RUL and not on predicting a simple point value.

By considering again the deterioration dynamics (14), the RUL control problem can be stated as follows: 
Problem 3. RUL control problem: At every time instant, find a control decision, denoted $\theta^{*}$ (i.e. $\theta^{*}$ could be any decision variable, for instance $\omega^{*}$ and/or $p\left(\omega^{*}\right)$ that drives the deterioration dynamics (14)), such that for a given set of current possible deterioration conditions $\alpha, m$ and $D$, the predicted RUL satisfy the (desired) requirements despite the presence of uncertainties on the current states, uncertainties on the deterioration dynamics (14) and its parameters.

In this paper, Problem 1 will be solved by using an Extended Kalman Filter which allows us to estimate the current deterioration state and its uncertainties. For the solution of Problem 2, we will use those estimations as initial conditions for predicting the RUL, and according to this prediction, for re-planning the trajectories of the driven device by choosing a more suitable trajectories for $\omega *$, by solving the Problem 3.

This paper focuses on the proposition and the development of a methodological solution for Problem 1, and shows how this solution can then be used to solve Problem 2. Bu we will also show how Problem 3 can be solved by using the proposed solution of Problems 1 and 2 and we propose an overall architecture for its solution. The proposed solutions are finally illustrated through a numerical example.

\section{Deterioration estimation of the friction drive}

\subsection{Extended system model for deterioration estimation}

Consider the following augmented system, obtained from dynamics (13) and (14):

$$
\begin{aligned}
\dot{\omega} & =A(\alpha) \omega+B u \\
\dot{\alpha} & =-m p(\omega) \alpha+D \varepsilon \\
\dot{m} & =\varepsilon \\
\dot{D} & =p(\omega) \alpha
\end{aligned}
$$

with:

$$
\begin{gathered}
A(\alpha):=\left[\begin{array}{cc}
\left(-\alpha r_{1}^{2}-B_{1}\right) / J_{1} & \alpha r_{1} r_{2} / J_{1} \\
\alpha r_{2} r_{1} / J_{2} & \left(-\alpha r_{2}^{2}-B_{2}\right) / J_{2}
\end{array}\right], \\
B=\left[\begin{array}{c}
1 / J_{1} \\
0
\end{array}\right]
\end{gathered}
$$


The input $u$ concerns the motor torque $T_{m}$ and the input $\varepsilon$ stands for unknown endogenous disturbances producing changes on $m$. It is assumed that the only available measurements are the physical variables (drive speeds $\omega$ ). That is

$$
y=\left[\begin{array}{l}
\omega_{1} \\
\omega_{2}
\end{array}\right]+\left[\begin{array}{l}
\eta_{1} \\
\eta_{2}
\end{array}\right]
$$

A nonlinear state-space representation of the model can be written in a compact form as follows:

$$
\begin{aligned}
& \dot{\boldsymbol{x}}=\boldsymbol{H}(\boldsymbol{x}) \boldsymbol{x}+\boldsymbol{B} u+\boldsymbol{E}(\boldsymbol{x}) \varepsilon \\
& \boldsymbol{y}=\boldsymbol{C} \boldsymbol{x}+\eta
\end{aligned}
$$

with

$$
\begin{aligned}
\boldsymbol{H}(\boldsymbol{x}) & =\left[\begin{array}{ccccc}
\frac{\left(-\alpha r_{1}^{2}-B_{1}\right)}{J_{1}} & \frac{\alpha r_{1} r_{2}}{J_{1}} & 0 & 0 & 0 \\
\frac{\alpha r_{2} r_{1}}{J_{2}} & \frac{\left(-\alpha r_{2}^{2}-B_{2}\right)}{J_{2}} & 0 & 0 & 0 \\
0 & 0 & 0 & -p(\omega) \alpha & 0 \\
0 & 0 & 0 & 0 & 0 \\
0 & 0 & p(\omega) & 0 & 0
\end{array}\right], \\
\boldsymbol{B} & =\left[\begin{array}{c}
1 / J_{1} \\
0 \\
0 \\
0 \\
0
\end{array}\right], \quad \boldsymbol{C}=\left[\begin{array}{lllll}
1 & 0 & 0 & 0 & 0 \\
0 & 1 & 0 & 0 & 0
\end{array}\right],
\end{aligned}
$$

and

$$
\boldsymbol{E}(\boldsymbol{x})=\left[\begin{array}{c}
0 \\
0 \\
D \\
1 \\
0
\end{array}\right],
$$

where the state vector of the augmented system is now $\boldsymbol{x}:=\left[\omega_{1}(t) \omega_{2}(t) \alpha(t) m(t) D(t)\right]^{\top}$ and $u=T_{m}(t)$ is the known system input. 
The nonlinear system (23)-(24) can be observed by using an Extended Kalman Filter (EKF). In this work, we adopt this technique for providing both the state estimation and a metrics of its uncertainties (e.g. the error covariance matrix, denoted $\boldsymbol{P}_{k \mid k}$ in Fig. 4). Recent techniques for which metrics on the estimation uncertainty are provided could be used instead of the EKF, see for instance [22], where censored measurements and parameter uncertainties are considered.

\subsection{Synthesis of an Extended Kalman Filter}

Consider the system (23)-(24) where $\varepsilon$ and $\eta$ are assumed to be Gaussian process noise and measurement noise respectively, with zero mean and covariance matrices $Q$ and $R$ respectively.

Matrix $Q$ must to take into account neglected and/or unmodelled dynamics. Here, it is assumed that modeling errors are mainly due to poor knowledge of the $m$ dynamics. Matrix $R$ represents the assumption that both speed sensors are affected by measurement noise ; it is assumed that this noises are more important than modeling errors.

Assuming the availability of discrete-time measurements at every time-instant, with a sample time $t_{s}$, the a priori prediction of the state estimate can be calculated by using the following discrete-time model:

$$
\hat{\boldsymbol{x}}_{k \mid k-1}=\left(I_{n}+t_{s} \cdot \boldsymbol{H}\left(\hat{\boldsymbol{x}}_{k-1 \mid k-1}\right)\right) \hat{\boldsymbol{x}}_{k-1 \mid k-1}+\boldsymbol{B} u_{k-1}
$$

The prediction of the a priori error covariance matrix $\boldsymbol{P} \in \mathbb{R}^{5}$ is calculated at every time instant as:

$$
\boldsymbol{P}_{k \mid k-1}=\boldsymbol{F}_{k-1} \boldsymbol{P}_{k-1 \mid k-1} \boldsymbol{F}_{k-1}^{\top}+Q
$$

where $\boldsymbol{F}_{k-1}$ is the Jacobian of the function $\boldsymbol{H}(\boldsymbol{x}):=f(\boldsymbol{x}) \boldsymbol{x}$ in discrete time. That is, $\boldsymbol{F}_{k-1}=$ $I_{n}+t_{s} \cdot F$, with

$$
F=\left.\frac{\partial \boldsymbol{f}(\boldsymbol{x})}{\partial \boldsymbol{x}}\right|_{\hat{\boldsymbol{x}}_{k \mid k-1}}
$$

calculated as:

$$
\frac{\partial \boldsymbol{f}(\boldsymbol{x})}{\partial \boldsymbol{x}}=\left[\begin{array}{ccccc}
F_{11} & F_{12} & F_{13} & 0 & 0 \\
F_{21} & F_{22} & F_{23} & 0 & 0 \\
F_{31} & F_{32} & F_{33} & F_{34} & 0 \\
0 & 0 & 0 & 0 & 0 \\
F_{51} & F_{52} & F_{53} & 0 & 0
\end{array}\right]
$$


where $F_{11}=-\left(\alpha r_{1}^{2}+B_{1}\right) / J_{1}, F_{12}=\left(\alpha r_{1} r_{2}\right) / J_{1}, F_{13}=\left(r_{1} r_{2} w_{2}-r_{1}^{2} w_{1}\right) / J_{1}, F_{21}=\left(\alpha r_{1} r_{2}\right) / J_{2}$, $F_{22}=-\left(\alpha r_{2}^{2}+B_{2}\right) / J_{2}, F_{23}=\left(r_{1} r_{2} w_{1}-r_{2}^{2} w_{2}\right) / J_{2}, F_{31}=-2 \alpha m r_{1}\left(r_{1} w_{1}-r_{2} w_{2}\right), F_{32}=2 \alpha m r_{2}\left(r_{1} w_{1}-\right.$ $\left.r_{2} w_{2}\right), F_{33}=-m\left(r_{1} w_{1}-r_{2} w_{2}\right)^{2}, F_{34}=-\alpha\left(r_{1} w_{1}-r_{2} w_{2}\right)^{2}, F_{51}=2 \alpha r_{1}\left(r_{1} w_{1}-r_{2} w_{2}\right), F_{52}=$ $-2 \alpha r_{2}\left(r_{1} w_{1}-r_{2} w_{2}\right)$ and $F_{53}=\left(r_{1} w_{1}-r_{2} w_{2}\right)^{2}$.

The innovation covariance, denoted $\boldsymbol{S} \in \mathbb{R}^{2}$, is obtained as follows:

$$
\boldsymbol{S}_{k}=\boldsymbol{C} \boldsymbol{P}_{k \mid k-1} \boldsymbol{C}^{\top}+R
$$

and the Kalman Gain as:

$$
\boldsymbol{K}_{k}=\boldsymbol{P}_{k \mid k-1} \boldsymbol{C}^{\top} \boldsymbol{S}_{k}^{-1}
$$

The updating of the state estimate is then:

$$
\hat{\boldsymbol{x}}_{k \mid k}=\hat{\boldsymbol{x}}_{k \mid k-1}+\boldsymbol{K}_{k}\left(\boldsymbol{y}_{k}-\boldsymbol{C} \hat{\boldsymbol{x}}_{k \mid k-1}\right)
$$

Finally, the a posteriori covariance matrix can be updated with

$$
\boldsymbol{P}_{k \mid k}=\left(\boldsymbol{I}-\boldsymbol{K}_{k} \boldsymbol{C}\right) \boldsymbol{P}_{k \mid k-1}
$$

Then, the estimation process re-starts again (at every sampling time $t_{s}$ ), by considering all the updated and estimated state vectors and covariance matrices. The estimation process requires the initialization of the estimated state at instant $k=0$, and an initial a priori covariance matrix $\boldsymbol{P}_{0 \mid 0}$.

Let us define the state estimation error as

$$
\tilde{\boldsymbol{x}}_{k \mid k}:=\boldsymbol{x}_{k}-\hat{\boldsymbol{x}}_{k \mid k}
$$

Considering that the expected value of $\tilde{\boldsymbol{x}}_{k \mid k} \in R^{5}$ is equal to zero, its covariance equal to $\boldsymbol{P}_{k \mid k}$ and $\boldsymbol{c}>0$ any real number, one can use the multidimensional Chebyshev's inequality stated in [23] :

$$
\operatorname{Pr}\left(\tilde{\boldsymbol{x}}_{k \mid k}^{T} \boldsymbol{P}_{k \mid k}^{-1} \tilde{\boldsymbol{x}}_{k \mid k}>\boldsymbol{c}^{2}\right) \leq \frac{n}{\boldsymbol{c}^{2}}
$$

This inequality is very useful because it can be applied to any probability distribution of the estimation error $\tilde{\boldsymbol{x}}_{k \mid k}$ in which the mean and variance are defined. In this paper, we propose to use the inequality (37) to obtain an adequate initial a priori covariance matrix $\boldsymbol{P}_{0 \mid 0}$, as well as to obtain stochastic intervals for every state estimation over time. That is, at any instant, one can 
use (37) to establish an interval of possible values of the state $\boldsymbol{x}_{k}$ with a given probability.

Using geometrical properties of the ellipsoids, as proposed in [24], bounds on the estimation error $\tilde{\boldsymbol{x}}_{k \mid k}$, denoted $\overline{\boldsymbol{x}}_{k}$, can be obtained as follows ${ }^{1}$ :

$$
\overline{\boldsymbol{x}}_{k}=\operatorname{diag}\left(\boldsymbol{P}_{k \mid k}^{1 / 2}\right) \boldsymbol{c}
$$

and then, at any instant $k$, the actual state will belong to those intervals with a probability defined by the scalar $\boldsymbol{c}$. For instance, the following inequalities are met:

$$
\begin{aligned}
& \hat{\boldsymbol{x}}_{k \mid k}(1)-\overline{\boldsymbol{x}}_{k}(1) \leq \omega_{1} \leq \hat{\boldsymbol{x}}_{k \mid k}(1)+\overline{\boldsymbol{x}}_{k}(1) \\
& \hat{\boldsymbol{x}}_{k \mid k}(2)-\overline{\boldsymbol{x}}_{k}(2) \leq \omega_{2} \leq \hat{\boldsymbol{x}}_{k \mid k}(2)+\overline{\boldsymbol{x}}_{k}(2) \\
& \hat{\boldsymbol{x}}_{k \mid k}(3)-\overline{\boldsymbol{x}}_{k}(3) \leq \alpha_{k} \leq \hat{\boldsymbol{x}}_{k \mid k}(3)+\overline{\boldsymbol{x}}_{k}(3) \\
& \hat{\boldsymbol{x}}_{k \mid k}(4)-\overline{\boldsymbol{x}}_{k}(4) \leq m_{k} \leq \hat{\boldsymbol{x}}_{k \mid k}(4)+\overline{\boldsymbol{x}}_{k}(4) \\
& \hat{\boldsymbol{x}}_{k \mid k}(5)-\overline{\boldsymbol{x}}_{k}(5) \leq D_{k} \leq \hat{\boldsymbol{x}}_{k \mid k}(5)+\overline{\boldsymbol{x}}_{k}(5)
\end{aligned}
$$

with a probability greater than $1-\left(n / \boldsymbol{c}^{2}\right)$. Here $n=5$ because $\boldsymbol{x} \in R^{5}$. That means that with $\boldsymbol{c}=10$ one can expect that the real value is within the previous intervals with a probability higher than $95 \%$. In the context of an EKF, this conclusion is true only if there are no modeling errors and the disturbances are properly characterized into the matrices $Q$ and $R$. The latter can be verified by checking the consistence of the innovations. This point will be discussed in the next subsection.

Remark that for a given expected initial bounds $\overline{\boldsymbol{x}}_{0}$ and scalar $\boldsymbol{c}$, an a priori initial covariance matrix $\boldsymbol{P}_{0 \mid 0}$ can be computed using (38). That is, the matrix $\boldsymbol{P}_{0 \mid 0}$ could be a diagonal matrix which verifies:

$$
\overline{\boldsymbol{x}}_{0}=\operatorname{diag}\left(\boldsymbol{P}_{0 \mid 0}^{1 / 2}\right) \boldsymbol{c}
$$

\subsection{Checking the consistence of the innovations}

In practice one can not measure the performance of the observer with respect to the state error measures, since there is no knowledge of the true values of the states. Hence, one can check if the observer is performing correctly in terms of the innovation covariance $\boldsymbol{S}$.

\footnotetext{
${ }^{1}$ In this paper, for any symmetric positive definite matrix $M \in \mathbb{R}^{n \times n}$, the matrix $M^{1 / 2} \in \mathbb{R}^{n \times n}$ is the elementwise square-roots of matrix $M$. The function $\operatorname{diag}(M)$ provides the diagonal of a square matrix $M$.
} 


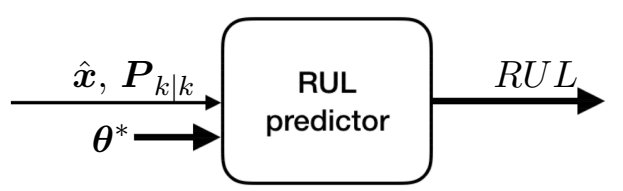

Figure 2: RUL predictor system. The inputs are the estimated deterioration state, its uncertainties and a decision vector $\boldsymbol{\theta}^{*}$ concerning desired (or expected) future operation conditions. The output concerns the predicted RUL distribution.

It is known that if the observer is working correctly then $\tilde{\boldsymbol{e}}_{k}:=\boldsymbol{y}_{k}-\boldsymbol{C} \hat{\boldsymbol{x}}_{k \mid k-1}$ is a white noise with zero mean and a covariance $\boldsymbol{S}_{k}$. Thus, one can verify that the observer is consistent by applying the following two procedures: i) check that the innovations are consistent with their covariance, and ii) check that the innovations are unbiased and white noise.

Because it is assumed that the measurement noise covariance matrix $R$ is well known, the only degree of freedom for tuning the EKF becomes the matrix $Q$. This matrix has to be chosen in such a way that the consistence of the innovation are verified. That is, the computed variance of every element of $\tilde{\boldsymbol{e}}_{k}$ has to be smaller than those estimated by the innovation covariance matrix $\boldsymbol{S}$.

\section{Prediction and control of the Remaining Useful Life}

\subsection{Prediction of the Remaining Useful Life}

Consider the decision vector $\boldsymbol{\theta}^{*}$, as depicted in Fig. 2. Vector $\boldsymbol{\theta}^{*}$ stands for a (possible) sequence of proposed motion characteristics influencing $\omega$, and then $p(\omega)$. We suppose that the sequence $\boldsymbol{\theta}^{*}$ will be optimally generated by a RUL controller. From Fig. 2, remark that one of the important inputs of the RUL predictor concerns the estimated state (angular velocities and the deterioration states) and a measure of their uncertainties. This means that the RUL prediction is mainly subject to the estimated state and the corresponding co-variance matrix estimation coming from a state estimator. The precision of the prediction clearly depends on the precision of the state estimation, as it is depicted in the conceptual Fig. 3. As shown in this figure, for the RUL prediction, the current state of deterioration and its uncertainties are used for creating several 
realizations of the deterioration trajectories by performing Monte Carlo simulations: at the time of prediction, realizations of the deterioration level and of the deterioration model parameter are drawn randomly within the estimation uncertainty interval (according to the estimated normal law) and a deterioration trajectory starting from each deterioration level realization is propagated until the failure. These trajectories and the corresponding failure times allow us to obtain an expected RUL distribution, that can be used in turn for control purposes.

Suppose now that the operation conditions are a priori planned as a function of one of the physical states. For instance, consider a motion control problem concerning the speed of the driven device $\omega_{2}$. Note that the original function $p(\omega)$ could be written, from (3) and (5), in terms of the mechanical trajectories on $\omega_{2}$ and the state of the contact surfaces $\alpha$. That is,

$$
p(\omega) \equiv p\left(\omega_{2}, \dot{\omega}_{2}, \alpha\right)=\left(\frac{B_{2} \omega_{2}+J_{2} \dot{\omega}_{2}}{\alpha r_{2}}\right)^{2}
$$

This parameterization simplifies the proposed deterioration model and can be used during the prediction stage. Then, the RUL predictor will perform predictions by considering the desired mechanical trajectories on $\omega_{2}$, denoted $\omega_{2}^{*}$, by using (45) and the deterioration model (14). Thus, the proposed RUL predictor performs simulations based on the following dynamics:

$$
\left[\begin{array}{c}
\dot{\alpha} \\
\dot{m} \\
\dot{D}
\end{array}\right]=\left[\begin{array}{c}
-m \alpha \\
0 \\
\alpha
\end{array}\right] p\left(\omega_{2}^{*}, \alpha\right)+\left[\begin{array}{c}
D \\
1 \\
0
\end{array}\right] \varepsilon
$$

For controlling the ratio of deterioration and consequently controlling RUL, the decision variable $\boldsymbol{\theta}^{*}$ could be a vector including optimal "future" positive or negative increments on $\omega_{2}^{*}$. This is because the ratio of deterioration (see the dynamics of $D$ in (46)), monotonically increases or decreases for positive or negative increments on $\omega_{2}^{*}$. For instance, into the numerical example, we will perform positive or negative increments on $\omega_{2}^{*}$ by using a scalar decision variable $\boldsymbol{\theta}^{*}=\theta^{*}$ as follows:

$$
\omega_{2}^{*}(\theta)=\omega_{2}^{r e f}+\theta^{*}
$$

where $\omega_{2}^{r e f}$ will concern the exogenous wheel speed reference used as an input of the motion controller. Hence, in this paper, RUL prediction will be performed by using model (46) affected by the input (47). 


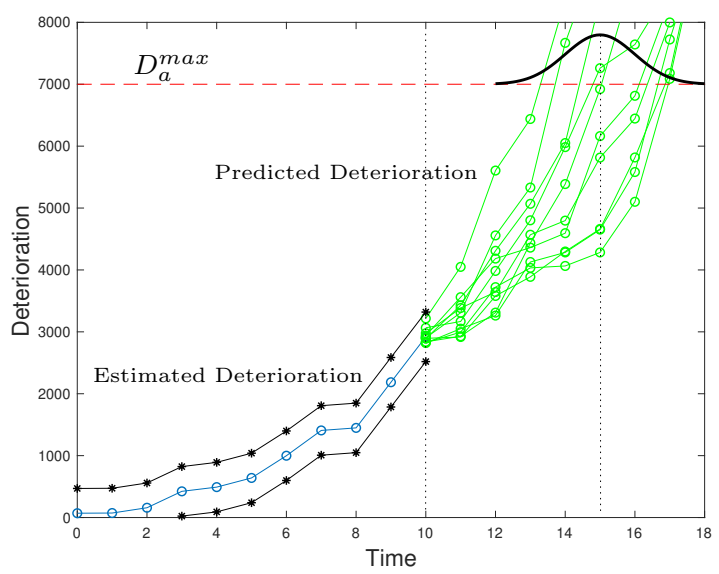

Figure 3: State of deterioration and its corresponding predicted trajectories for obtaining expected RUL distribution by using Monte Carlo simulation.

\subsection{A control principle of the Remaining Useful Life}

The proposed control architecture, depicted in Fig. 4, includes a standard motion controller and a RUL controller based on the solution of an iterative optimization problem. The motion controller is intended for respecting motion requirements, whereas the RUL controller is designed for solving a stochastic optimisation problem due to the random nature of the RUL. That RUL controller provides decisions using $\theta^{*}$ that properly modify the motion references (used for the motion controller) to ensure a desired RUL. The RUL controller is based on RUL predictions and has to ensure compliance with the motion requirements but also with the desired RUL. Thus, $\theta^{*}$ could concern a parameter of the motion controller as it is proposed in [25]. In [25] the control architecture allows controlling the RUL by suitable filtering the desired motor torques but without considering the motion controller. In this work, we assume that the motion controller can be written as a function of the mechanical states, the motion references (considered as a known exogenous input) but also the RUL control decision variable $\theta^{*}$. That is, the motor torque can be written as follows:

$$
T_{m}=T_{m}\left(\omega, \omega_{r e f}, \theta^{*}\right)
$$

For instance, into the numerical example, the motor torque provided by the motion controller at the instant $k$ will be the following standard proportional-integral motion controller:

$$
T_{m}(k)=-c_{1}\left(\omega_{2}-\omega_{2}^{r e f}-\theta^{*}\right)-c_{2} \int_{0}^{t}\left(\omega_{2}-\omega_{2}^{r e f}-\theta^{*}\right) d t
$$


where $\theta^{*}$ explicitly appears into the motion control law and can be interpreted as a dynamical adaptation of the speed references. The constants $c_{1}$ and $c_{2}$ are suitable designed for respecting motion tracking requirements. Now, due to the fact that the predicted RUL is a random variable, the RUL controller design problem can be stated as a stochastic optimization problem. That is, given the state estimations $\hat{\boldsymbol{x}}_{k \mid k}$ and a measure of their uncertainties $\boldsymbol{P}_{k \mid k}$, coming from the state estimator at a time-instant $k$, find $\theta^{*}$ that minimizes an objective cost function $\mathcal{L}$, i.e. such that

$$
\theta^{*}:=\underset{\theta \in \mathbb{R}}{\arg \min } \mathcal{L}(\theta)
$$

subject to

$$
\operatorname{Pr}\left(R U L\left(\hat{\boldsymbol{x}}_{k \mid k}, \boldsymbol{P}_{k \mid k}, \theta\right) \leq R U L^{r e f}\right) \leq \bar{\eta}
$$

where $\bar{\eta}$ and $R U L^{\text {ref }}$ concerns desired (decision) parameters.

The function $\mathcal{L}(\theta)$ could include all functions characterizing the desired motion requirements. For instance, if we are interested in a speed tracking problem this function could be:

$$
\mathcal{L}(\theta):=\mathcal{L}\left(\omega(\theta)-\omega^{r e f}\right)=\frac{1}{2}\left(\omega_{2}^{*}(\theta)-\omega_{2}^{r e f}\right)^{2}=\frac{1}{2} \theta^{2}
$$

which penalizes the size of the positive or negative increments on the desired wheel speed, $\omega_{2}^{*}(\theta)$, defined in (47). Therefore, the optimization problem is now to find the smallest positive or negative increments which guarantee the respect of the constraint (51).

As presented in [26], this kind of stochastic optimization problems, (50)-(51), can be re-stated in a more tractable one by enumerating a finite number of admissible solutions $\theta$ and solving the problem using randomized methods, see for instance [27]. In the next section, due to the fact that the predicted RUL monotonically increases or decreases with $\theta$, the numerical example is performed by considering the following iterative optimization:

$$
\theta^{*}(s+1):=\theta^{*}(s)+\mathcal{K}\left(R U L-R U L^{r e f}\right)
$$

where $\mathcal{K}\left(R U L-R U L^{r e f}\right)$ is a function which provides positive or negative increments by considering the difference between the predicted RUL and the desired one. The value $\theta^{*}(s)$ concerns the current decision, and the $\theta^{*}(s+1)$ the successor decision, at a time-instant $s$.

The location of the proposed deterioration estimator, for predicting and controlling RUL of a friction drive system, is depicted on the control architecture in Fig. 4. 


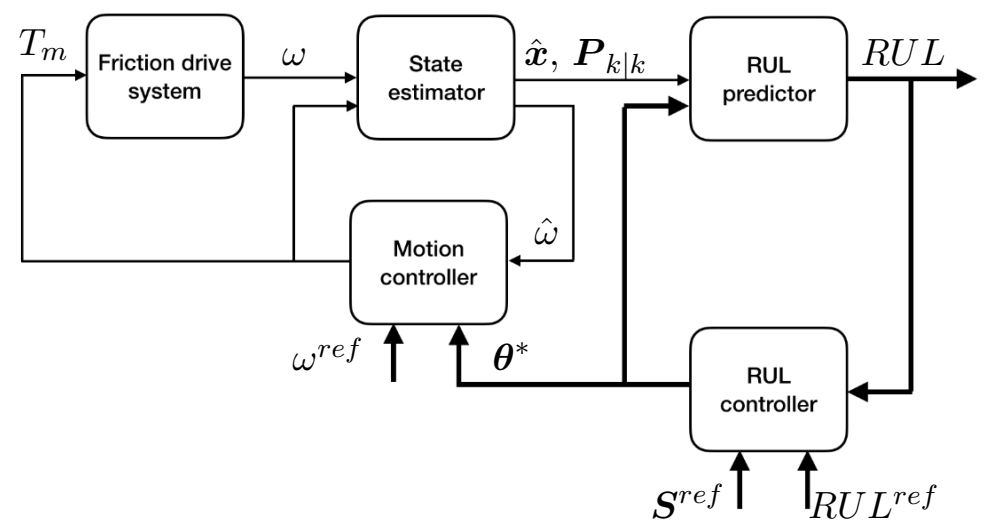

Figure 4: Control architecture for controlling actuator motion and RUL.

Table 1: Constant parameters of the Friction drive system

\begin{tabular}{lll}
\hline Symbol & Value & Units \\
\hline$r_{1}$ & 0.0315 & {$[\mathrm{~m}]$} \\
$r_{2}$ & 0.35 & {$[\mathrm{~m}]$} \\
$B_{1}$ & $6.36 \mathrm{e}-3$ & {$\left[\mathrm{Kgm}^{2} / \mathrm{s}\right]$} \\
$B_{2}$ & 0.6250 & {$\left[\mathrm{Kgm}^{2} / \mathrm{s}\right]$} \\
$J_{1}$ & $9.9225 \mathrm{e}-04$ & {$\left[\mathrm{Kgm}^{2}\right]$} \\
$J_{2}$ & 12.25 & {$\left[\mathrm{Kgm}^{2}\right]$} \\
\hline
\end{tabular}

In Fig. 4, the decision vector $\theta^{*}$ will be imposed to the motion controller in order to guarantee a desired behavior for $\omega$ given $\omega^{r e f}$. The input $\boldsymbol{S}^{r e f}$ is used here in order to explicitly include any motion satisfaction criteria and/or to capture desired trade-offs for the multi-objective optimization problem that has to be solved by the RUL controller.

\section{A numerical example}

In this section, an example of a friction drive system described by equations (13) and (14), with parameters given in Table 1, is considered. An Extended Kalman Filter is used as a state estimator including deterioration estimation as proposed in Section 4 . The chosen matrix $R$ is: 


$$
R=\left[\begin{array}{cc}
33.33 & 0 \\
0 & 0.33
\end{array}\right],
$$

which assumes that the sensor noise variances are known and equal to 33.33 and 0.33 , for sensor 1 and 2 respectively. The matrix $Q$ has been chosen such that consistence of innovations is obtained, as explained in Section 4.3, which supposes the availability of data from the estimation process that can be used for re-tuning matrix $Q$. This re-tuning can be performed during the first sample times, as recently proposed in [28]. Remember that, in order to reach the consistence of innovations, the actual innovation variances have to be smaller or equal to those provided by the estimated innovation covariance matrix computed using 32 .

The matrix $Q$ has been selected as follows, which provides consistence of innovations:

$$
Q=\left[\begin{array}{ccccc}
0.1 & 0 & 0 & 0 & 0 \\
0 & 0.001 & 0 & 0 & 0 \\
0 & 0 & 0 & 0 & 0 \\
0 & 0 & 0 & 0 & 0 \\
0 & 0 & 0 & 0 & 0
\end{array}\right]
$$

In this example, we assume that the initial conditions of system states are unknown but bounded, and the corresponding bounds are known. Thus, the initial conditions are assumed to be bounded as follows:

$$
\hat{\boldsymbol{x}}_{0 \mid 0}-\overline{\boldsymbol{x}}_{0} \leq \boldsymbol{x}_{0} \leq \hat{\boldsymbol{x}}_{0 \mid 0}+\overline{\boldsymbol{x}}_{0}
$$

with

$$
\hat{\boldsymbol{x}}_{0 \mid 0}=\left[\begin{array}{c}
0 \\
0 \\
11 \\
1.1 e-6 \\
0
\end{array}\right], \overline{\boldsymbol{x}}_{0}=\left[\begin{array}{c}
23 \\
2 \\
5 \\
1 e-6 \\
5 e 5
\end{array}\right]
$$


and then, the initial matrix $\boldsymbol{P}_{0 \mid 0}$, computed using (44) with $c=10$, will be

$$
\boldsymbol{P}_{0 \mid 0}=\left[\begin{array}{ccccc}
5.29 & 0 & 0 & 0 & 0 \\
0 & 0.04 & 0 & 0 & 0 \\
0 & 0 & 0.25 & 0 & 0 \\
0 & 0 & 0 & 1 e-14 & 0 \\
0 & 0 & 0 & 0 & 2.5 e-9
\end{array}\right]
$$

The EKF performs state estimations as illustrated in Figures 5, 6 and 7. As described in Section 4.2, for any probability distribution law of each estimate, the parameter $c=10$ allows us to conclude that there exists a possibility that the actual state is outside the intervals with a probability smaller or equal to $5 \%$, see inequality (37).

In this example, the motor torque provided by the motion controller is:

$$
T_{m}(t)=-0.0048\left(\omega_{2}-\omega_{2}^{r e f}-\theta^{*}\right)-0.0048 \int_{0}^{t}\left(\omega_{2}-\omega_{2}^{r e f}-\theta^{*}\right) d t
$$

\subsection{Estimation scenario : description and analysis}

In order to illustrate the relevance of the proposed EKF for estimating the deterioration states and the parameter of the deterioration model, the following scenario concerning the mechanical states trajectories has been considered.

Estimation Scenario: The motion controller sets a torque such that the driven device follows a reference speed $\omega_{2}^{r e f}=20 \mathrm{rad} / \mathrm{s}$ (for $\theta^{*}=0$, nominally). During the whole useful life of the actuator this reference speed is maintained constant. The parameter $m$ has been modeled as a constant parameter during the EKF design, however this parameter changes abruptly from $m=1 e-6$ to $m=2 e-6$ at time $t=4$ hours, increasing the speed of deterioration.

Figures 5, 6 and 7 depict the obtained results for the considered estimation scenario. Due to the deterioration, $\omega_{2}=0$ (which corresponds to a complete deterioration) has been reached before 8 hours of system life. From Figure 5, it is possible to remark that the motion controller increases the speed $\omega_{1}$ in order to maintain the driven device speed $\omega_{2}$ around $20 \mathrm{rad} / \mathrm{s}$ as much as possible. Remark also that before reaching the complete deterioration, and without the use of a deterioration model and an associated filtering procedure, it is almost impossible to ascertain the presence of 
deterioration, looking directly at the behavior of $\omega_{1}$ and/or $\omega_{2}$. This is because, in one hand $\omega_{2}$ remains constant and follows the desired reference, and on the other hand, the increments on the speed $\omega_{1}$ could be interpreted "in practice" as increments due to a possible augmentation of the mechanical loads at the driven side.

Regarding the deterioration model parameter and deterioration states estimated from the proposed EKF, the parameters $\alpha$ and $m$ are properly estimated, even if the estimations are provided with different levels of precision (size of intervals). The uncertainties on $\alpha$ and $m$ decrease as more data is available in time. Note also that the obtained estimations clearly highlight the presence of deterioration. The parameter $\alpha$ decreases and $D$ increases in such a way that it can be concluded that the parameter $m$ is constant. However, after 4 hours of system life, an abrupt change appears on $m$. The EKF cannot follow this change because $m$ is considered as a constant parameter within the used model. The EKF instead provides a value of the estimated $m$ that deviates from the previously calculated mean value.

Re-starting the EKF. In order to complement the proposed estimation procedure with a capacity to track changes in the parameter of the deterioration model, let suppose that there exists a supervisor system able to determine that the behavior of the estimated $m$ is not consistent with the assumption on that parameter. The EKF can be re-started by considering a change on the expected bounds on $\alpha$ and $m$ due to the detected model error. This is achieved through a re-initialization of the matrix $\boldsymbol{P}_{k \mid k}$ by a matrix which satisfies (38) under the novel system conditions. Figure 6 illustrates such a re-initialization and re-starting of the EKF and its effect on the estimation of $\alpha$ and $m$. The re-initialization is performed at around 4.3 hours of the system life, after a delay for the detection of the abrupt change that occurred at 4 hours.

\subsection{Prediction scenarios : description and analysis}

In order to gain a better insight into the behavior of the proposed RUL prediction scheme based on the estimated deterioration, several "prediction scenarios" are explored : scenarios with and without re-starting the EKF after the detection of an abrupt change of the $m$ parameter, a scenario with an example of RUL control and finally a scenario where $m$ is taken random. 

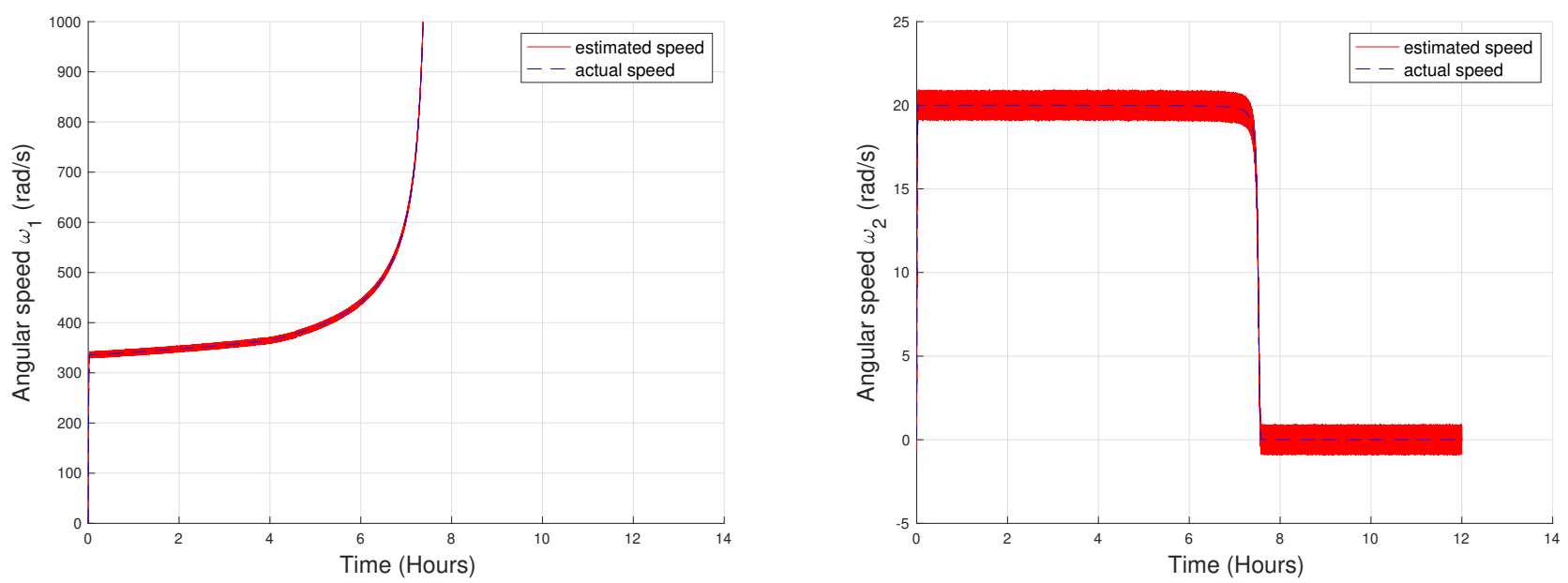

Figure 5: Estimation scenario: the angular speeds $\omega_{1}$ and $\omega_{2}(\mathrm{in} \mathrm{rad} / \mathrm{s})$ are estimated along the whole useful life of a given friction drive by using noisy measurements. The system lifetime is less than 8 hours, and $\omega_{2}=0$ after reaching the maximal deterioration.
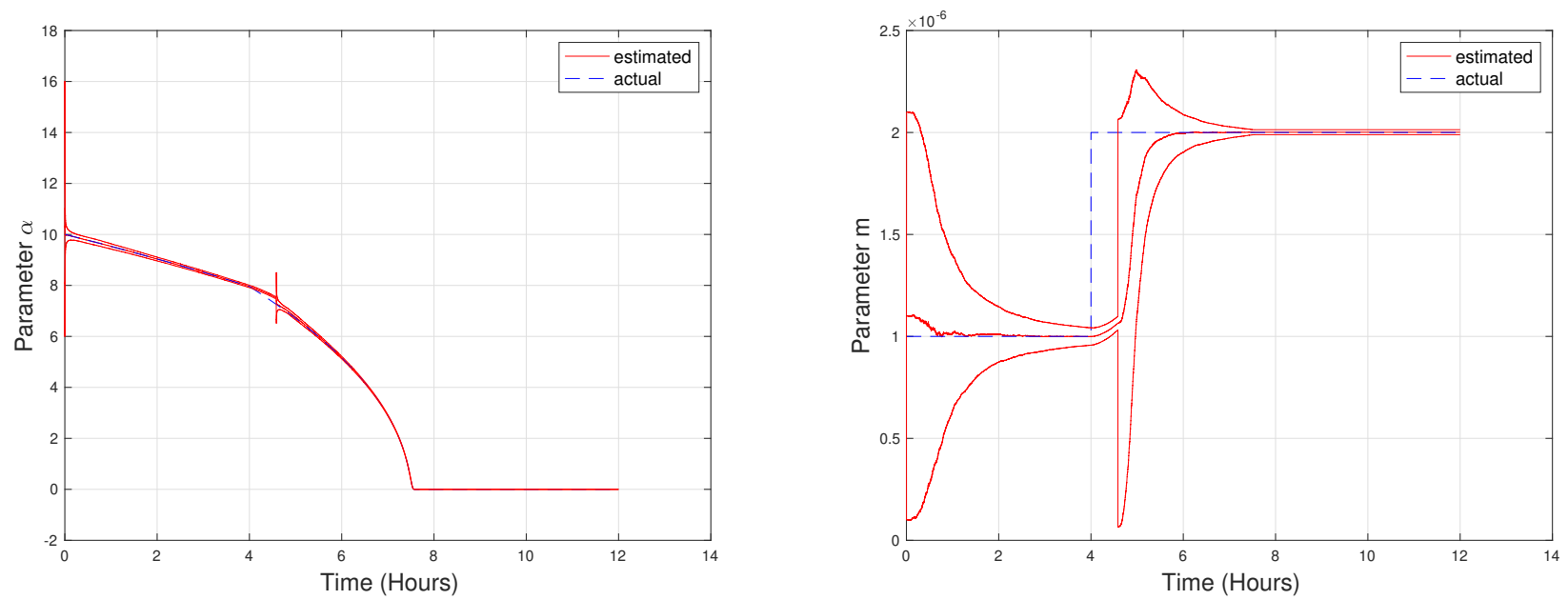

Figure 6: Estimation scenario: the parameters $\alpha, m$ and their associated uncertainty intervals are estimated along the whole useful life of a given friction drive. After detection of an abrupt change on $m$, which is not considered within the initial model, the EKF observer is re-started with a new matrix $\boldsymbol{P}_{0 \mid 0}$ at around 4.3 hours. 


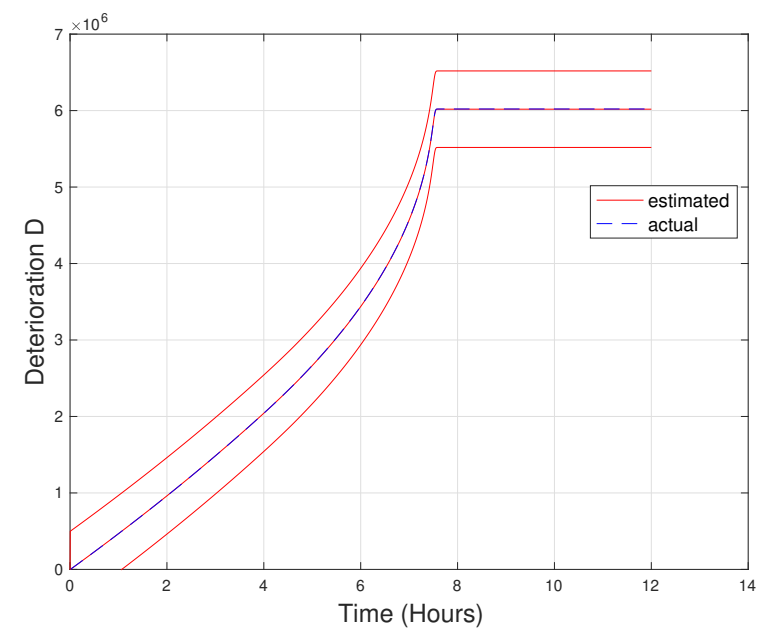

Figure 7: Estimation scenario: the deterioration level $D$ and its uncertainty interval are estimated along the whole useful life of a given friction drive.

Prediction Scenario 1 (without re-starting the $E K F$ ). The state estimator performs estimations as described in the estimation scenario presented in Section 6.1. However, just before a time equal to 4.3 hours a RUL prediction is performed without considering that estimations of parameters $m$ and $\alpha$ are no longer true, as depicted in Figures 8 and 9. In particular, RUL predictions do not consider that assumptions concerning $m$ (i.e. $\dot{m}=0$ ) are not longer met. Due to this difference between the assumed model on the evolution of $m$ and its actual behavior, the obtained mean for the predicted end of life of the system (EoL) is around 9.09 hours, whereas the real value is less than 8 hours, as shown in Figure 9.

Prediction Scenario 2 (with re-starting the $\mathbf{E K F}$ ). The state estimator performs estimations as described in the estimation scenario presented in Section 6.1. Just after a time equal to 5.0 hours a RUL prediction is performed. The RUL predictor considers the new estimated values concerning the parameter $m$, and performs predictions based on the assumption that $\dot{m}=0$. However, the RUL controller has not yet updated the optimal value of $\theta^{*}$, and consequently the speed $\omega_{2}$ is maintained around $20 \mathrm{rad} / \mathrm{s}$. Figures 10 and 11 illustrate this scenario. The mean of the predicted system EoL is now around 7.55 hours which corresponds to a more accurate prediction, thanks to the re-starting procedure of the EKF. 
Prediction Scenario 3 (with re-starting the EKF and performing RUL control). The state estimator performs estimations as described in the estimation scenario presented in Section 6.1. Just after a time equal to 5.0 hours a RUL prediction is performed. The RUL predictor considers the new estimated values concerning the parameter $m$, and performs predictions based on the assumption that $\dot{m}=0$. The RUL controller has updated the optimal value of $\theta^{*}$, and then the speed $\omega_{2}$ is constrained to follow a value around $16 \mathrm{rad} / \mathrm{s}$. This modification of $\theta^{*}$ and of the desired speed $\omega_{2}$ leads to a modification of the deterioration behavior, which in turn modify the system lifetime. The updated EKF integrating the new value of $\theta^{*}$ is able to provide a new EoL estimation which corresponds to the desired one (e.g. respecting condition (51)). Figures 12 and 13 illustrate this scenario : they show how a suitable change in the value of the decision variable $\theta^{*}$ provides EoL values that satisfy the given specifications, and how this new controlled EoL can be predicted by the updated EKF. The mean of the controlled EoL is around 9.0 hours and most of the trajectories present a total useful life that is more than 8.0 hours.

Prediction Scenario 4 (with re-starting the EKF and random parameter $m$ ). The state estimator performs estimations as it is described in the estimation scenario. However, here it is considered that the actual parameter $m$ is a random variable with a normal distribution. The proposed deterioration estimator follows the behavior of this parameters despite its noisy behavior. Remark that the model used for the observer synthesis remains unchanged. Figure 14 illustrates the behavior of the obtained estimations on $m$. Hence, the deterioration model (14) is general enough to integrate different sources of randomness. The Figure 15 illustrates the behavior of the predictions on $\alpha$ and $D$, which remain accurate. 

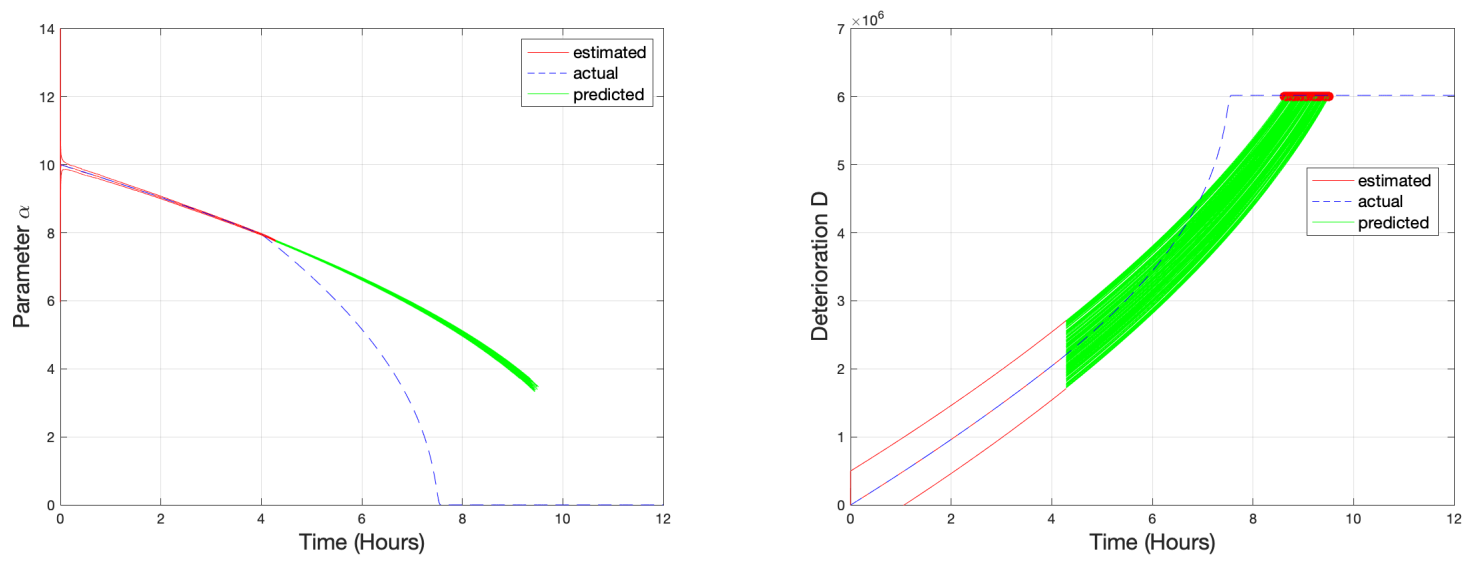

Figure 8: Prediction scenario 1: estimation of $\alpha$ (left) and $m$ (right) without correction of the estimation process.
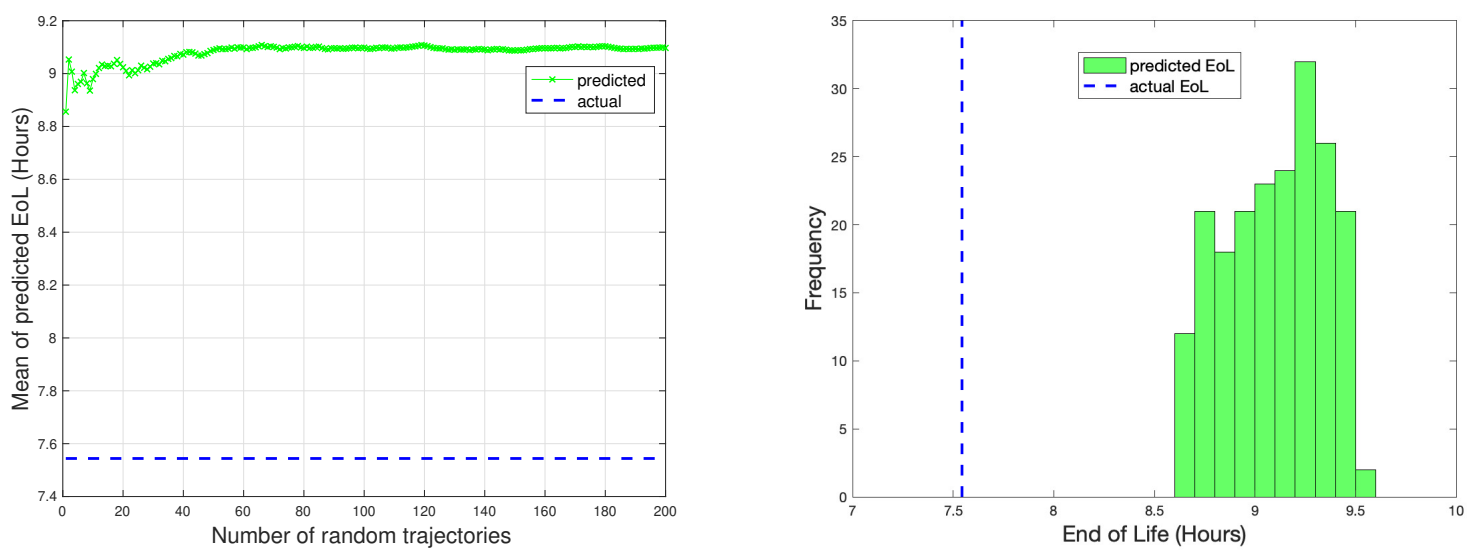

Figure 9: Prediction scenario 1: mean and histogram of the predicted system end of life (EoL) without correction of the estimation process. 

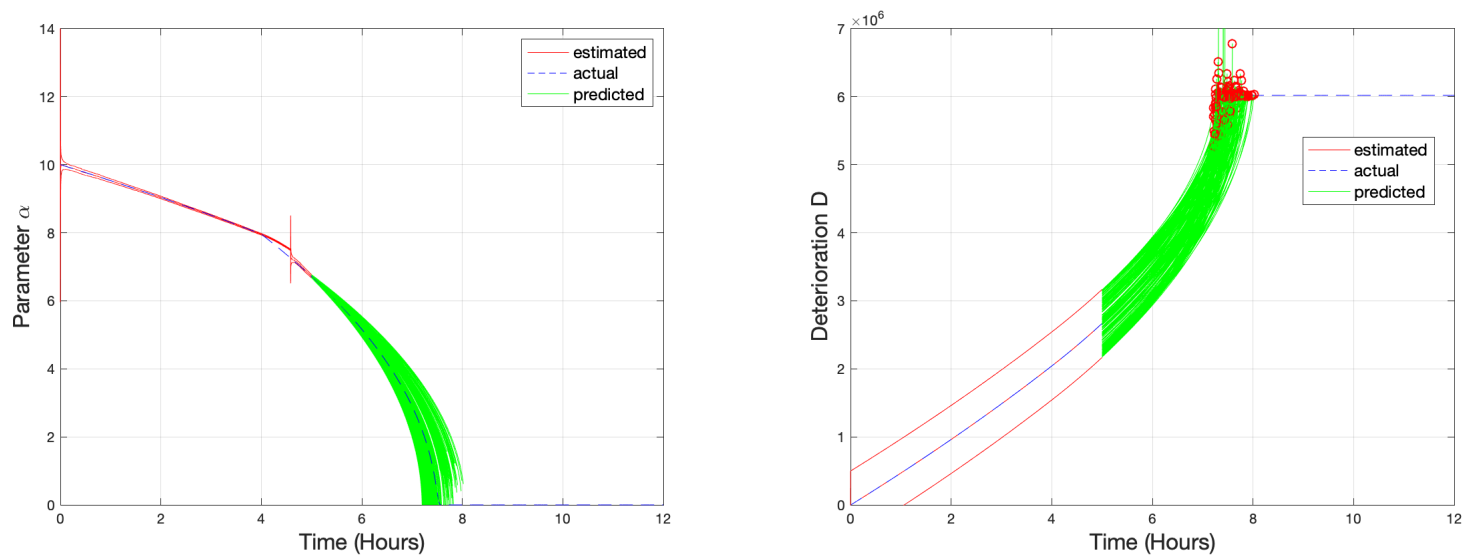

Figure 10: Prediction scenario 2: estimation of $\alpha$ (left) and $m$ (right) with correction of the estimation process.
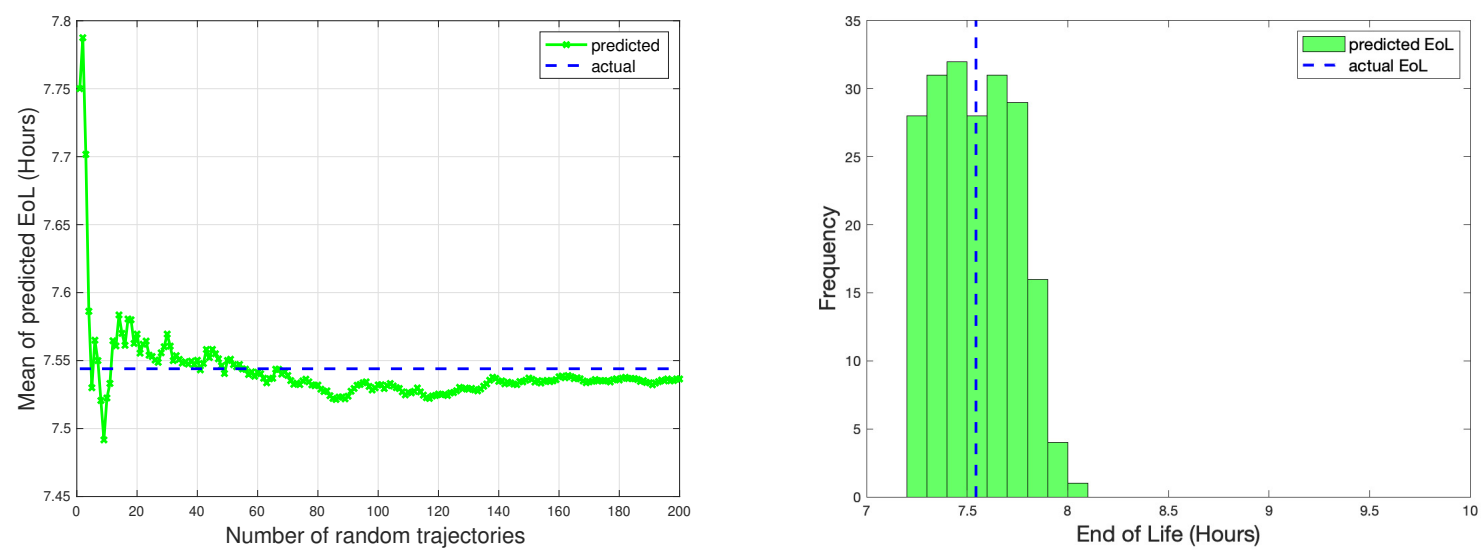

Figure 11: Prediction scenario 2: mean and histogram of the predicted system end of life (EoL) with correction of the estimation process. 

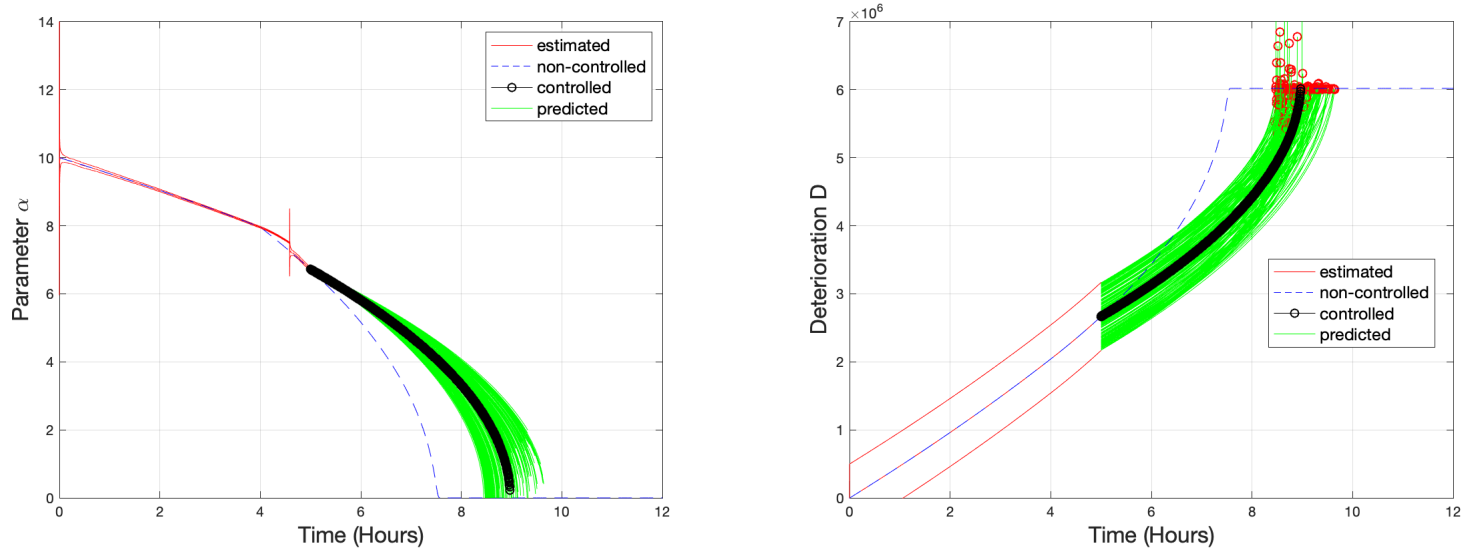

Figure 12: Prediction scenario 3: Evolution of $\alpha$ and $m$ (controlled vs. non controlled, estimated and predicted)

- With correction of the estimation process.
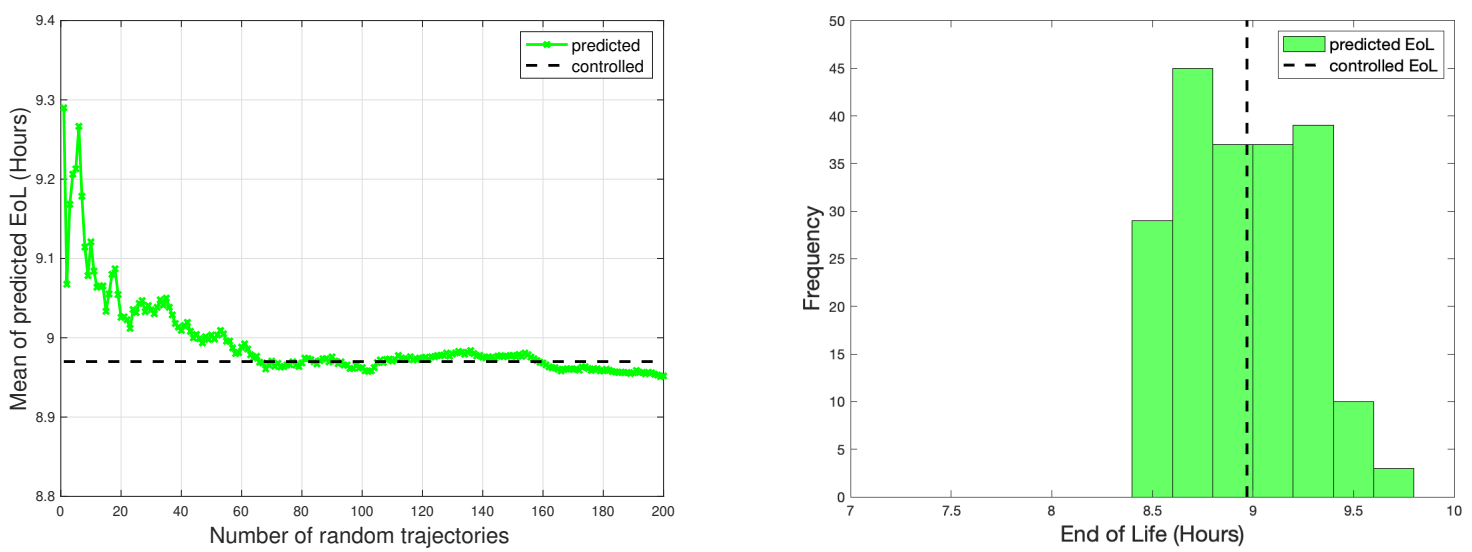

Figure 13: Prediction scenario 3: mean and histogram of the predicted and controlled system end of life (EoL) With correction of the estimation process. 


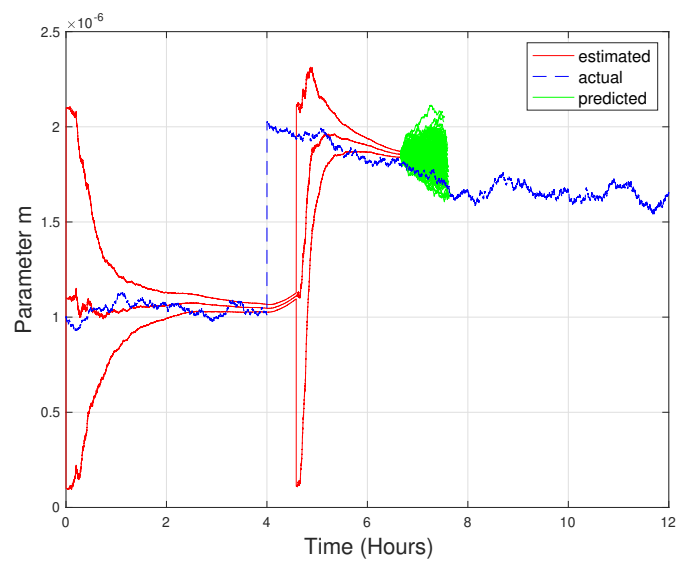

Figure 14: Prediction scenario 4: Estimated and predicted trajectories of parameter $m$ when taken as random following a normal distribution with mean into the estimated uncertainty interval (only 10 predicted trajectories of $m$ are shown for a better readability)

\section{Conclusions}

Within this paper a method has been presented for performing an online deterioration estimation a friction drive system, which can be used in turn for prediction and control of the Remaining Useful Life of the system. In the absence of the possibility of a direct (or even indirect measurement) of the deterioration (or of a covariate correlated with it), the originality of the approach is to resort to an extended dynamical system model, including both system motion and deterioration dynamics, for estimating the current deterioration state based on external measurements of physical quantities (here the angular speeds) that are easily accessible. Due to the non-linear nature of the system dynamics, an Extended Kalman Filter (EKF) has been proposed for obtaining both an estimation of the state vector and its associated uncertainties. Within this work an integrated architecture for RUL prognosis and control is also proposed, in which the control decision is elaborated using the online estimated deterioration with its associated uncertainties. Thanks to the used physics-based deterioration model, the approach for the RUL control is based on the possibility of acting on physical variables (e.g. re-configuring motor torques and/or making adjustments to the reference inputs, i.e. desired angular speed) to modify the deterioration behavior, which in turn allows to control indirectly the RUL in closed-loop, based on RUL prognosis information. The implementation and the performance of the deterioration estimator have been illustrated through a numerical example which includes different scenarios of prediction and control of the Remaining 

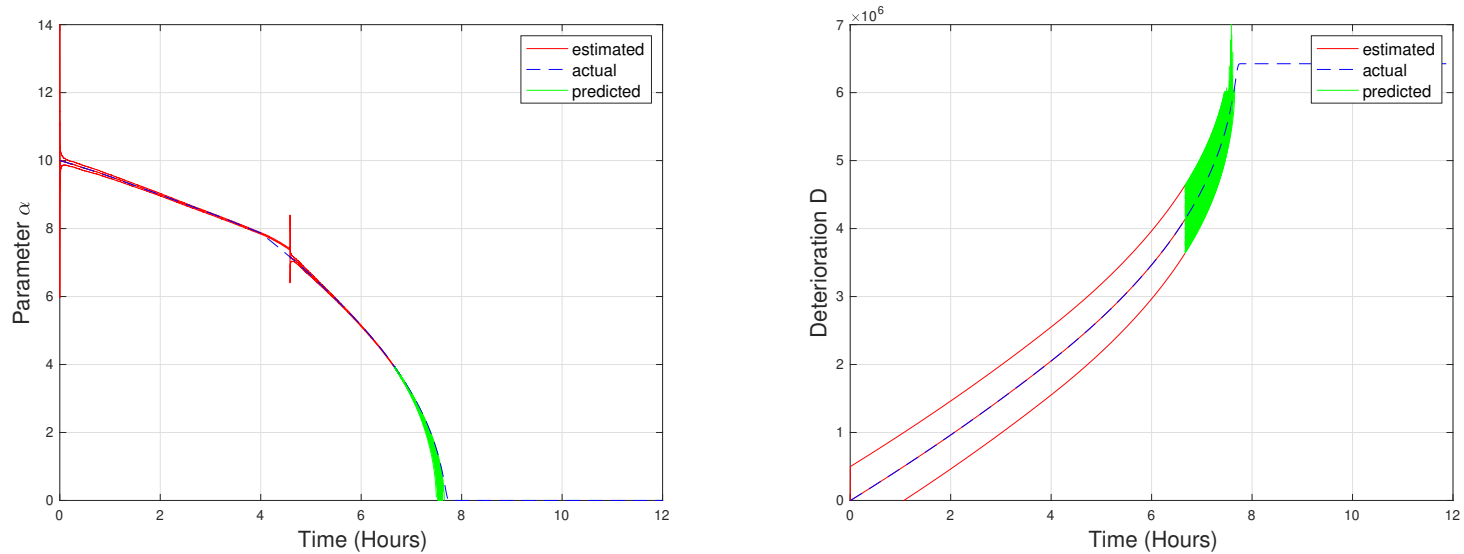

Figure 15: Prediction scenario 4: Deterioration ( $\alpha$ and $D)$ with correction of the estimation process when $m$ is taken as random following a normal distribution with mean into the estimated uncertainty interval (only 10 predicted trajectories of $m$ are shown for a better readability)

Useful Life for such devices ; these numerical experiments have shown specifically how the prediction and the control of RUL could be improved by a suitable re-starting the EKF, allowing to use the knowledge about the system functioning and the available real-time data coming from sensors and actuators.

The framework proposed in this paper can be seen as a first methodological step towards the development of an integrative scheme for deterioration estimation, RUL prognosis and RUL control, based on "external", directly and easily available monitoring information. Our future research work will be to further investigate control algorithms aiming to adapt the operation of the system to its online estimated deterioration and reliability so as to better control its lifetime balancing it with its performance level, hence developing a reliability-aware control system.

\section{Acknowledgments}

This work was partially supported by the French CNRS PEPS 2017 in the context of the IMPROVED project.

\section{References}

[1] T. Meyer, C. Sondermann-Wölke, J. Kimotho, W. Sextro, Controlling the remaining useful lifetime using selfoptimization, Chem. Eng. Trans 33 (2013) 625-630. 
[2] T. Meyer, W. Sextro, Closed-loop control cystem for the reliability of intelligent mechatronic systems, in: Proc. of PHME 2014 - 2nd European Conference of the Prognostics and Health Management Society, 2014, pp. 53-57.

[3] V. Atamuradov, K. Medjaher, P. Dersin, B. Lamoureux, N. Zerhouni, Prognostics and health management for maintenance practitioners - Review, implementation and tools evaluation, International Journal of Prognostics and Health Management 8 (7) (2017) 31 p.

[4] H. Skima, C. Varnier, E. Dedu, K. Medjaher, J. Bourgeois, Post-prognostics decision making in distributed MEMS-based systems, Journal of Intelligent Manufacturing 30 (3) (2019) 1125-1136.

[5] D. W. Brown, G. Georgoulas, B. Bole, H. L. Pei, M. Orchard, L. Tang, B. Saha, A. Saxena, K. Goebel, G. Vachtsevanos, Prognostics enhanced reconfigurable control of electro-mechanical actuators, in: Proc. of PHM 2009 - Annual Conference of the Prognostics and Health Management Society, 2009, pp. 1-7.

[6] A. Bogdanov, S. Chiu, L. U. Gökdere, J. Vian, Stochastic optimal control of a servo motor with a lifetime constraint, in: Proc. of 45th IEEE Conference on Decision and Control, IEEE, 2006, pp. 4182-4187.

[7] E. Balaban, J. J. Alonso, A modeling framework for prognostic decision making and its application to uav mission planning, in: Proc. of PHM 2013 - Annual Conference of the Prognostics and Health Management Society, 2013, pp. 449-460.

[8] E. Balaban, J. J. Alonso, An approach to prognostic decision making in the aerospace domain, in: Proc. of PHM 2012 - Annual Conference of the Prognostics and Health Management Society, 2012, pp. 396-415.

[9] S. Sankararaman, K. Goebel, Why is the Remaining Useful Life prediction uncertain?, in: Proc. of PHM 2013 - Annual Conference of the Prognostics and Health Management Society, 2013, pp. 337-349.

[10] B. Bole, L. Tang, K. Goebel, G. Vachtsevanos, Adaptive load-allocation for prognosis-based risk management, in: Proc of PHM 2011 - Annual Conference of the Prognostics and Health Management Society, 2011, pp. 67-76.

[11] Y. Langeron, A. Grall, A. Barros, Joint maintenance and controller reconfiguration policy for a gradually deteriorating control system, Proceedings of the Institution of Mechanical Engineers, Part O: Journal of Risk and Reliability 231 (4) (2017) 339-349.

[12] D. Nguyen, L. Dieulle, A. Grall, Remaining Useful Lifetime prognosis of controlled systems: A case of stochastically deteriorating actuator, Mathematical Problems in Engineering 2015 (2015) 16p.

[13] M. A. Cremona, B. Liu, Y. Hu, S. Bruni, R. Lewis, Predicting railway wheel wear under uncertainty of wear coefficient, using universal kriging, Reliability Engineering \& System Safety 154 (2016) 49-59.

[14] D. J. Rodriguez Obando, J. J. Martinez Molina, C. Berenguer, Deterioration estimation for remaining useful lifetime prognosis in a friction drive system, in: Proc. of IFAC 2017 - 20th World Congress of the International Federation of Automatic Control, 2017, pp. 13324-13329.

[15] D. J. Rodriguez Obando, J. J. Martinez Molina, C. Berenguer, Condition monitoring of a friction drive system, in: Proc. of 3rd IEEE Colombian Conference on Automatic Control, 2017, p. 6p.

[16] K. Astrom, C. Canudas-de-Wit, Revisiting the LuGre friction model, IEEE Control Systems Magazine 28 (6) (2008) 101-114.

[17] C. Canudas-de-Wit, P. Tsiotras, E. Velenis, M. Basset, G. Gissinger, Dynamic friction models for road/tire longitudinal interaction, Vehicle System Dynamics 39 (3) (2003) 189-226.

[18] J. Martinez, J. Avila, C. Canudas-de-Wit, A new bicycle vehicle model with dynamic contact friction, in: IFAC 
Symposium on Advances in Automotive Control, 2004, pp. 625-630.

[19] D. J. Rodriguez Obando, J. J. Martinez Molina, C. Berenguer, Deterioration modelling of contact surfaces for a friction drive system, in: Proc. of the 26th European Safety and Reliability Conference- ESREL 2016, 2016, pp. 1961-1968.

[20] A. Bevan, P. Molyneux-Berry, B. Eickhoff, M. Burstow, Development and validation of a wheel wear and rolling contact fatigue damage model, Wear 307 (2013) 100-111.

[21] S. Mercier, W. Kahle, C. Paroissin, Degradation Processes in Reliability, Wiley-ISTE, 2016.

[22] C. Huang, B. Shen, H. Chen, H. Shu, A dynamically event-triggered approach to recursive filtering with censored measurements and parameter uncertainties, Journal of the Franklin Institute 356 (15) (2019) 8870 - 8889.

[23] B. Stellato, B. V. Parys, P. Goulart, Multivariate Chebyshev inequality with estimated mean and variance, The American Statistician 71 (2) (2017) 123-127.

[24] J. J. Martinez, N. Loukkas, N. Meslem, H-infinity set-membership observer design for discrete-time LPV systems, International Journal of Control (2018) 1-12.

[25] D. J. Rodriguez Obando, J. J. Martinez Molina, C. Berenguer, An architecture for controlling the remaining useful lifetime of a friction drive system, in: IFAC PapersOnline - Proc. 10th IFAC Symposium on Fault Detection, Supervision and Safety for Technical Processes - SAFEPROCESS 2018, Vol. 51, 2018, pp. 861-866.

[26] M. Alamir, On probabilistic certification of combined cancer therapies using strongly uncertain models, Journal of Theoretical Biology 384 (2015) 59-69.

[27] T. Alamo, R. Tempo, E. F. Camacho, Randomized strategies for probabilistic solutions of uncertain feasibility and optimization problems, IEEE Transactions on Automatic Control 54 (11) (2009) 2545-2559.

[28] J. Gonzalez Silva, J. O. De Aquino, E. Liborio, Adaptive extended kalman filter using exponencial moving average, IFAC-PapersOnLine 51 (25) (2018) 208 - 211, proc. 9th IFAC Symposium on Robust Control Design ROCOND 2018. 